\title{
Quadriceps Tendon Rupture due to Postepileptic Convulsion
}

Mehmet Sabri Balik, MD, Adem Erkut, MD, Yilmaz Guvercin, MD, Rifat Sahin, MD, and Davut Keskin, MD

Department of Orthopaedic and Traumatology, Recep Tayyip Erdogan University School of Medicine, Rize, Turkey

We present a case of quadriceps tendon (QT) rupture. QT ruptures can occur in all ages. The cause is mostly traumatic in origin. Spontaneous ruptures that are thought to result from predisposing conditions are rare. Post-convulsion QT ruptures lacking traumas in their history can be overlooked in clinical examinations. This should be born in mind by the attending physician, as early diagnosis and treatment of the condition can lead to satisfactory outcomes.

Keywords: Knee joint, Quadriceps,Tendon injuries, Epileptic seizures

A quadriceps tendon (QT) rupture can be encountered in all age groups. It generally occurs after low energy trauma, such as falling down, in elderly patients and after high energy trauma, such as sport injuries, in young patients ${ }^{1}$. Non-traumatic ruptures of the QT are rare. In spontaneous QT ruptures, local and metabolic factors play a role as predisposing factors, such as secondary hyperparathyroidism, beta-microglobulin associated amyloidosis, corticosteroid treatment and fluoroquinolone use ${ }^{2-4)}$. In addition, long-term usage of anti-epileptic medication (AEM) weakens adhesion of the tendon to the bone ${ }^{5}$. During seizures, already weakened tendon-bone insertion facilitates rupture ${ }^{6}$. Non-traumatic QT ruptures can be missed on physical examination. Herein, we want to emphasize that QT ruptures can develop after epileptic convulsion, which must be born in mind by the medical staff.

Received July 25, 2013; Revised (1st) September 17, 2013;

(2nd) November 14, 2013; Accepted February 27, 2014

Correspondence to: Mehmet Sabri Balik, MD

Department of Orthopaedic and Traumatology, Recep Tayyip Erdogan University School of Medicine, Islampasa Mahallesi, Sehitler Caddesi,

No: 74, 53100, Rize, Turkey

Tel: +90-464-213-0491, Fax: +90-464-212-3015

E-mail: sabribalik@yahoo.com

This is an Open Access article distributed under the terms of the Creative Commons Attribution Non-Commercial License (http://creativecommons.org/licenses/by-nc/3.0/) which permits unrestricted non-commercial use, distribution, and reproduction in any medium, provided the original work is properly cited.

\section{Case Report}

A 39-year-old male patient was admitted to our clinic with a complaint of inability to extend his right knee after an epileptic convulsion that occurred at rest. He had no history of trauma. The patient had a history of left patellar tendon (PT) rupture from the patellar insertion during previous epileptic seizures at rest and underwent two operations in the previous two years. The proximal part of the PT was ruptured from the insertion area of the patella on his presentation.

The interface of the bone and the ruptured tendon were refreshed. Then, tunnels were carved in the patella from the distal to proximal direction. Krackow suture was used to fix the proximal end of the PT. The free ends of the sutures were guided into the tunnels. The proximal part of the PT was tensely tightened. In this way, the PT was secured to the region of the patella where it had ruptured from. No complications were observed during the two- month follow-up. The knee regained full range of motion. Muscle power, pain score, and knee function were evaluated according to the Knee Society Clinical Rating System (KSCRS). The score was excellent 2 months after the operation.

Active extension of the right knee was absent. Swelling and tenderness were detected in the supra-patellar region. Additionally, a gap was also detected by palpation, which occurred due to the displacement of the ruptured tendon endings.

The hematologic parameters (white blood cell count, hemoglobin, platelet count, creatinin, uric acid, alkaline phosphatase, 


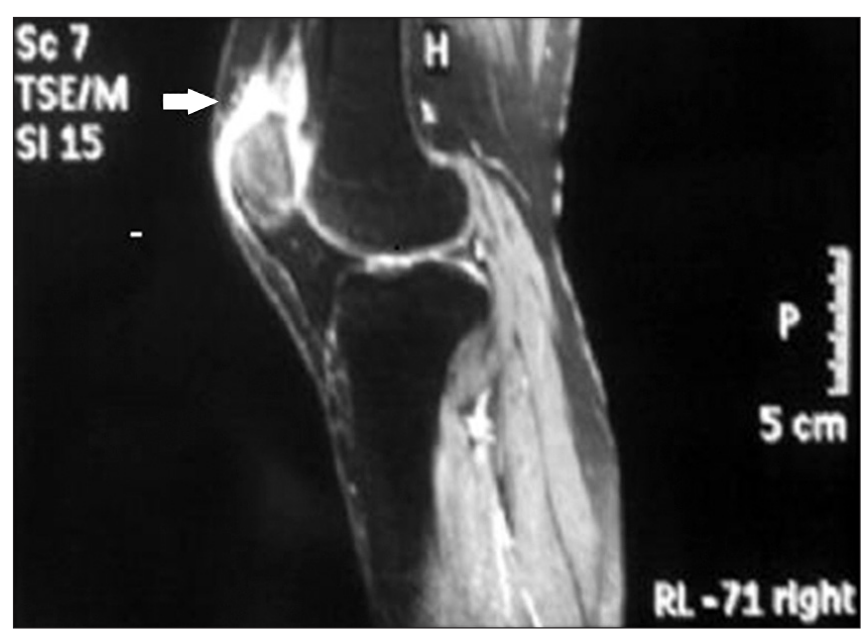

Fig. 1. Preoperative sagittal magnetic resonance image of the ridht knee showing complete rupture at the distal quadriceps tendo-osseous junction (arrow).

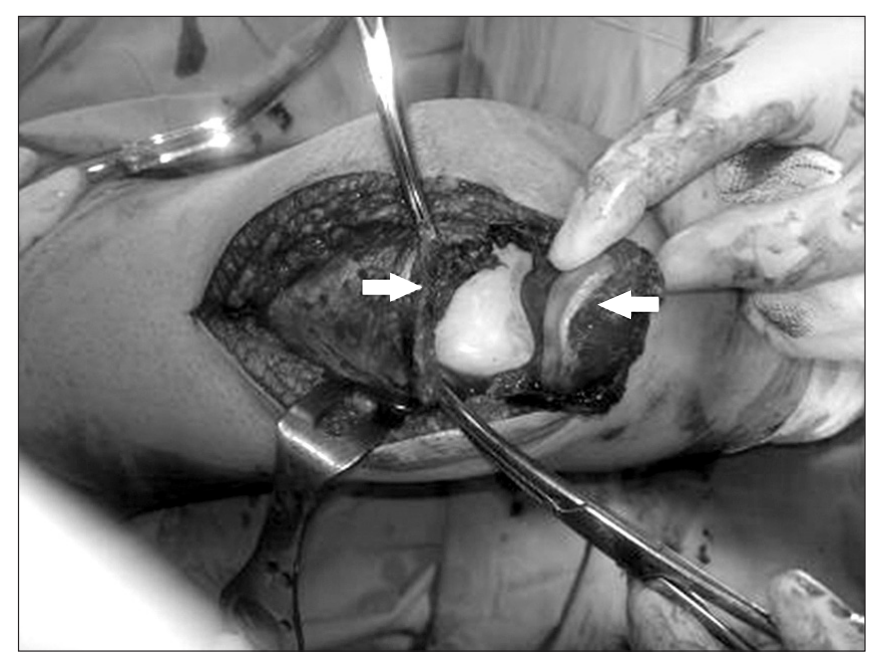

Fig. 2. Intraoperative appearance of the ruptured quadriceps tendon (arrows).

parathyroid hormone, calcium, phosphorus, and $25-\mathrm{OH}$ vitamin D) were within normal ranges.

The patient had a history of AEM use for 29 years.

Disruption of the QT was detected by magnetic resonance imaging (MRI) (Fig. 1). The patient was operated on the second day of hospitalization. The distal part of the QT was reached by a vertical incision. The distal part was ruptured from the insertion area of the upper patellar region (Fig. 2). The bone surface and the ruptured surface of the tendon were refreshed. Then, tunnels were carved in the patella from proximal to distal direction. Krackow suturing was performed in the distal QT (Fig. 3). The free ends of the sutures were guided through these tunnels.

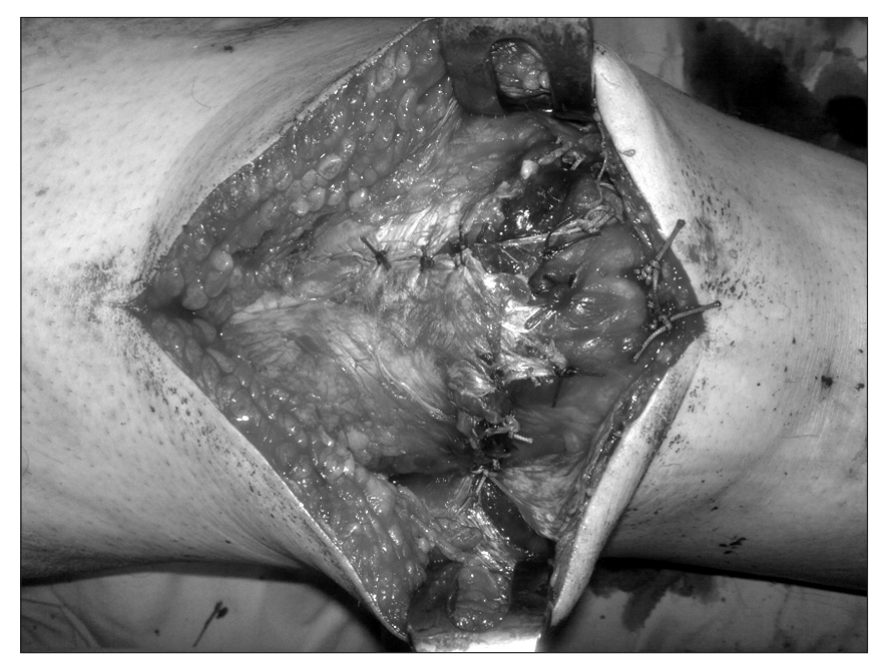

Fig. 3. Ruptured quadriceps tendon was sutured using a Krackow technique.

The distal part of the QT was tied up with tension. During this procedure, the QT was tied up to the upper region of the patella where it was detached from. A leg-length splint was applied for 2 weeks after the operation. A leg-length-tube cast was applied after removal of the sutures, which allowed active movements of the ankle, for 6 weeks. Afterwards, physiotherapy for muscle strengthening and improvement of range of motion of the knee was initiated. The patient had no complaints three months after the operation.

The knee regained full range of motion. Muscle power, pain score, and knee function were evaluated according to the KSCRS. The score was excellent 2 months after the operation.

\section{Discussion}

Bone fracture and ankle dislocation frequently occur during epileptic convulsions. The prevalence of post-epileptic convulsion bone fracture ranges between $0.25 \%$ and $2.4 \%{ }^{7)}$. However, there are a limited number of reports on QT ruptures after epileptic convulsion in the literature.

Predisposing factors for non-traumatic QT ruptures are described as follows ${ }^{2,3}$ : systemic disorders, such as lupus erythematosus; medications, such as long term steroid use; and micro traumas due to intensive sport activities ${ }^{2}$. In addition, long-term epileptic medication causes bone resorption ${ }^{5}$. During seizures, weakened tendon insertions are susceptible to ruptures. Even though traumas sustained as a result of falls during epileptic seizures can cause ruptures, ${ }^{5,6)}$, our patient had seizure at rest.

New injuries can occur in $1 / 3$ of epileptic patients. Awareness of 
possible new post-convulsion injuries is required by the medical community $^{7)}$. In our case, PT rupture was preceded by the QT rupture in the other extremity by five months.

Long-term AEM usage ( $>2$ years) is very likely to be the predisposing factor in our case. The patient had been on AEM for 29 years. The clinical and laboratory findings were normal and thus failed to point to any other likely causes. On the other hand, it has been shown that long-term AEM use may affect bone quality ${ }^{5-7)}$ by interfering with the gastrointestinal absorption of calcium ${ }^{7}$. Because these drugs can affect bone turn-over, bone mineral metabolism, peripheral activated vitamin $\mathrm{D}$, liver enzymes that assist in the metabolism of vitamin $\mathrm{D}$, osteomalacia may occur eventually ${ }^{7,8)}$.

The QT rupture in our case developed during convulsion without trauma. Strong tonic muscular contractions occur during epileptic convulsions. These strong muscular contractions rarely lead to locomotor system injuries ${ }^{4)}$. Therefore, it is possible that weakening of the bones due to prolonged use of AEM can lead to non- traumatic injuries during seizures.

A patient with QT rupture is unable to do active knee extension. There is swelling and pain in the ruptured area. Additionally, there is also a gap in the supra-patellar region ${ }^{9)}$. Imaging techniques can be used for confirming the clinical diagnosis. Swelling of the soft tissue in the supra-patellar area can be detected by standard radiography. MRI can also be used for confirming the diagnosis with an advanced radiological test. Early surgical intervention is necessary to ensure functional recovery in QT ruptures $^{3,10)}$. Loss of knee function, proximal retraction of the QT tendon, and patella baja are indicators of late diagnosis of QT ruptures ${ }^{10)}$.

$\mathrm{He}$ and associates followed 23 patients with QT rupture for 6 years. Early intervention groups had better results than the late intervention groups ${ }^{9}$. Additionally, other studies suggest that satisfactory results can be obtained by early diagnosis and early repairing of QT rupture ${ }^{2-4)}$.

Physicians may overlook non-traumatic post-convulsion QT ruptures in epileptic patients, in whom timely diagnosis and treatment can lead to satisfactory outcomes. We believe that this case report can be useful to achieve this purpose.

\section{Conflict of Interest}

No potential conflict of interest relevant to this article was reported.

\section{References}

1. La S, Fessell DP, Femino JE, Jacobson JA, Jamadar D, Hayes C. Sonography of partial-thickness quadriceps tendon tears with surgical correlation. J Ultrasound Med. 2003;22:1323-9.

2. Lu HD, Cai DZ, Wang K, Zeng C. Simultaneous bilateral patellar tendon rupture without predisposing systemic disease or steroid use: a case report. Chin J Traumatol. 2012;15:54-8.

3. Kim BS, Kim YW, Song EK, Seon JK, Kang KD, Kim HN. Simultaneous bilateral quadriceps tendon rupture in a patient with chronic renal failure. Knee Surg Relat Res. 2012;24:569.

4. Barasch E, Lombardi LJ, Arena L, Epstein E. MRI visualization of bilateral quadriceps tendon rupture in a patient with secondary hyperparathyroidism: implications for diagnosis and therapy. Comput Med Imaging Graph. 1989;13:407-10.

5. Chung S, Ahn C. Effects of anti-epileptic drug therapy on bone mineral density in ambulatory epileptic children. Brain Dev. 1994;16:382-5.

6. Vestergaard P. Epilepsy, osteoporosis and fracture risk: a meta-analysis. Acta Neurol Scand. 2005;112:277-86.

7. Friedberg R, Buras J. Bilateral acetabular fractures associated with a seizure: a case report. Ann Emerg Med. 2005;46:2602.

8. Vestergaard P. Changes in bone turnover, bone mineral and fracture risk induced by drugs used to treat epilepsy. Curr Drug Saf. 2008;3:168-72.

9. He L, Wang T, Wang M, Rong G. Patella ligament and femoral quadriceps tendon rupture. Zhonghua Wai Ke Za Zhi. 2002;40:918-22.

10. Al-Nakshabandi NA. Bilateral chronic quadriceps tendon tear secondary to repeated seizures. Neurosciences (Riyadh). 2006;11:115-6. 